\title{
A quantum Bell Test homodyne interferometer at ambient temperature for millimetre wave entangled photons
}

\author{
Neil A. Salmon \\ Manchester Metropolitan University, Manchester, M15 6BH, UK
}

\begin{abstract}
This paper investigates the feasibility of operating a Bell Test for millimetre wave entangled photons using ambient temperature instrumentation, raising the question as to whether this can be done in a regime where the photon energy ( $h f / e \sim 0.06$ milli-eV at $10 \mathrm{GHz}$ ) is far smaller than the thermal energy ( $k T / e \sim 25$ milli-eV at $290 \mathrm{~K}$ ). It also raises the question as to whether it is possible to generate entangled photons at these frequencies, as previously this has only been achieved using cryogenically cooled Josephson junctions. A homodyne interferometric receiver is proposed whereby a millimetre wave pump generates entangled photons by spontaneous parametric down-conversion in a non-linear birefringent material, before the signal and idler are mixed together in a sum-frequency mixer. The output then enters a second mixer which uses the pump as the local oscillator to shift the signal and idler down to baseband, where signal integration over many successive entangled pair recovers the entanglement signature from the noise. A successful demonstration of this would mean cryogenics could be avoided, enabling more sensor architectures and deployment scenarios. Novel experiments in the millimetre wave band could lead to a deeper understanding of entanglement and offer novel schemes for secure communications and covert interrogation techniques (quantum radar and ghost imaging), exploiting the fact that the signal is below the level of the thermal noise and can only be accessed by a single user having the key, which is the pump.
\end{abstract}

Keywords: Non-linear optics, quantum technology, entanglement, quantum mechanics, millimetre wave band, photons

\section{INTRODUCTION}

Since the introduction of entanglement [1] and a means to its validation (the Bell Test) was proposed [2], [3] a number of optical experiments have demonstrated the phenomenon [4], [5], [6]. These experiments have been conducted in and around the visible spectrum, because entanglement experiments are less difficult in this region than in other bands. This is because many non-linear materials are available in which entangled photons may be generated and relatively intense sources of spectrally pure radiation, by way of lasers, are available to pump this process. Furthermore, optical photon diode detectors have very high performance after many years of development in the optical communications industry. In addition the photon energy $(h f)$ in the visible band is much higher than the thermal energy $(k T)$, which puts the detection systems into the quantum regime. This means that there are virtually no thermally generated photons that would otherwise act as a noise source to degrade the system performance. The maturity of the optical technology means that the optical Bell Tests are a part of many undergraduate labs [7], loopholes in the violation of Bell's inequality have all but closed and a number of books on quantum optics [8], [9], [10], [11], [12] and quantum information theory [13] include sections on this research.

Moving into the millimetre wave band a small number of entanglement experiments have been developed which operate at cryogenic temperatures [14], [15]. Lowering the temperature is done to reduce the number of thermal photons which would otherwise degrade the system noise performance, so again this pushes the detection into the quantum regime.

Since operating at cryogenic temperature limits the number of deployment scenarios, it is worth considering if there is a means operating in the millimetre wave band without the inconvenience of cooling. This means operating in the Rayleigh-Jeans regime where the photon energy is much smaller than the thermal energy. As a result there will be many thermally generated photons, which will act as a source of noise. These are likely to swamp a relatively weak entangled photon flux. To address this issue a homodyne interferometer is proposed that uses signal integration techniques to overcome the thermally generated noise. The following approach uses a semi-classical approach to evaluate and simulate the proposed homodyne interferometer.

* Correspondence: Email: n.salmon at mmu.ac.uk; Telephone: +44 7921172892 


\section{GENERATING ENTANGLED MILLIMETRE WAVE PHOTONS PAIRS}

The creation of pairs of entangled photons can come about through the process of spontaneous parametric down conversion (SPDC), whereby a single incident photon of frequency f seemingly vanishes and two photons, whose frequencies sum to that of the incident photon, appear in its place [11]. The process takes place in materials that have a high non-linear susceptibility; that is materials which have structures that generate a polarised electric field $\mathrm{P}(\mathrm{t})$ given by

$$
P(t)=\varepsilon_{0}\left[\chi^{(1)} E(t)+\chi^{(2)} E^{2}(t)+\chi^{(3)} E^{3}(t)+\cdots\right]
$$

where $\mathrm{E}(\mathrm{t})$ is the externally applied electric field and $\chi$ is the electrical susceptibility of the material and superscripts (1), (2), (3), ... refer to the linear, second order and third order components. The orders of the second and above only have effects when the electric field associated with the incident photon flux is large.

Since the 1980's Bell Tests have predominantly used lasers, emitting near-VU radiation, to generate the high electric fields and non-linear optical materials such as Beta-Barium Borate $\mathrm{BBO}\left(\mathrm{BaB}_{2} \mathrm{O}_{4}\right.$ or $\left.\mathrm{Ba}\left(\mathrm{BO}_{2}\right)_{2}\right)$ to generate nearinfrared entangled photon pairs [10]. In these experiments the UV emission is referred to as the pump radiation and the two photons are referred to (for historical reasons associated with parametric amplification) [10] as the signal photon and the idler photon. The signal is assumed to be that of the higher frequency and the idler that of the lower frequency, but this distinction is not generally retained when discussing SPDC. When the two photons have exactly the same frequency, they are referred to as degenerate photons.

To date, many entanglement experiments have been aimed mainly at closing loopholes in calculating the Bell inequality and maximising the distance between the signal and idler whilst maintaining entanglement. The BBO material has high susceptibility, as its molecule already has a permanent electric dipole moment, this being due to its non-centrosymmetric geometry. In the Bell Tests it is not just necessary to generate the entangled photons, but it is also required that they be directed in two different directions, without identifying their individual polarisations. This is done by exploiting the birefringence of the material. Birefringence is that material property whereby two orthogonal polarisations (eg. right hand and left hand circular polarisation, or perpendicular and parallel linear polarisation) have different refractive indices.

The properties of a material or device for the generation of entangled photons by SPDC are:

1) Large non-linear susceptibility or characteristic

2) Birefringence

These two properties enable essentially two different types of architectures to create streams of entangled photons. These are referred to as Type I (where the polarisations of the two entangled photons are the same, and opposite to that incident photon) and as Type II (where the polarisations of the two entangled photons are opposite, with only one of these being the same as the incident photon) [11].

Two important properties of entangled photons that are exploited in the proposed homodyne interferometer Bell Test are:

1) The conservation of energy, which states:

$$
\omega_{P}=\omega_{S}+\omega_{I}
$$

2) The conservation of momentum, which states:

$$
\phi_{P}=\phi_{S}+\phi_{I}
$$

where $\omega$ and $\phi$ refer to the angular frequency and phase respectively and the subscripts $\mathrm{P}, \mathrm{S}, \mathrm{I}$, refer to the pump, signal and idler photons.

The wave spatial (z) variation in phase is through exp $j k z$, where $k$ is wave number $(2 \pi / \lambda)$, related to the photon momentum $p \sim h / \lambda$ through the de Broglie relation. The temporal variation of the wave is through $\exp -j \omega t$, related to the photon energy $E \sim \hbar \omega$, by the Einstein relation.

For the generation of entangled photons in the millimetre wave band perhaps the easiest way to proceed is to reproduce what has been done in the optical band using the closest device counterparts in the millimetre wave band. An optimum frequency at which to conduct millimetre wave experiments might be $20 \mathrm{GHz}$ for the pump, as the costs of the components are relatively inexpensive compared to the higher frequencies. The pump frequency should perhaps be no 
lower than $20 \mathrm{GHz}(\lambda \sim 15 \mathrm{~mm})$ as longer wavelengths would make any demonstration equipment bulky and cumbersome.

In the millimetre wave band it is possible to generate high electric field coherent wave radiation at $20 \mathrm{GHz}$ by using a solid state amplifier to amplify the output from either a backward wave oscillator or a Gunn diode. Using this method it is relatively easy to generate up to 1 Watt of radiation. Much larger output powers could be generated from a wide range of tube devices, if this was deemed necessary.

Two materials that are recognised as having strong non-linear characteristics in the millimetre wave band are Lithium Niobate $\left(\mathrm{LiNbO}_{3}\right)$ and Gallium Arsenide (GaAs). These materials might therefore be used as the medium in which to generate the entangled photons. There are a wide variety of devices that are manufactured by the semiconductor industry using these materials. Their non-linear characteristics are exploited in the manufacture of mixers for receivers and upconverters or multipliers to generate higher frequency radiations for communications and as local oscillators for mixers. These materials are also birefringent, so they have the two necessary properties for the generation of a beam of entangled photons which could be split into a signal beam and an idler beam.

Further details of the architecture of the source are not given here as it is really more of an engineering task to use the material properties to reproduce the entangled photons beams. They could be either of Type I or the Type II and they would typically be constructed in some type of waveguide configuration or in free-space. The signal and receiver arms of the analyser might view the source at a variety of angles and polarisations to identify the optimum configuration for the potential generation of entangled photon pairs. It is only really necessary to say at this stage that such a source could be constructed. The detector circuit described in the following section would then be used to confirm if such a source were generating entangled mm-wave photon pairs.

\section{ANALYSING THE ENTANGLED MILLIMETRE WAVE PHOTON PAIRS}

\subsection{Optical Bell Test systems in the quantum regime}

In the classic Bell tests conducted in the optical band the signal and idler photons are detected separately in individual single-photon avalanche diode (SPAD) detectors. The photon, with an energy greater than the semiconductor bandgap, raises an electron from the valence band into the conduction band, which subsequently gets accelerated by a high reversely biased electric field and initiates an avalanche breakdown process. This amplifies the current associated with the single electron, a process necessary to increase the signal to noise ratio of the measurement. A quenching circuit, operating on a nano-second timescale, then reduces the reverse bias below the breakdown voltage, after which is it raises it again, so the detector is ready for the next photon. In the classic Bell Test the single photon outputs of the SPAD detectors are passed to a threshold circuit and then enter a coincident counter. The coincidence counter acts as an incoherent integrator to further increase signal to noise ratio of the measurement. The count numbers are then used to calculate the 'test statistic' $S$ parameter which is then used to determine if the Bell inequality has been infringed. Infringement of the Bell inequality confirms the existence of entangled photon pairs.

\subsection{Millimetre wave Bell Test concept in the Rayleigh-Jeans regime}

A millimetre wave Bell test system operating at ambient temperature has the difficulty that the photon energy ( $h f$ ) at $10 \mathrm{GHz}$ (for degenerate entangled photons from a pump at $20 \mathrm{GHz}$ ) is 0.041 milli-eV, whilst the thermal energy ( $k T$ ) is $\sim 25$ mill-eV at 290 Kelvin. This means there will be many thermally excited photons which will swamp the entangled photons. For example, the number of photons in a single mode is given [12] by the Bose-Einstein mean occupation number,

$$
\bar{n}=\frac{1}{e^{h f / k T}-1}
$$

which in the quantum regime $(h f>k T)$ the becomes $\exp (-h f / k T)$, a very small number $\left(<2 \times 10^{-22}\right)$ in the visible band. However in the Rayleigh-Jeans regime, the mean photon occupation number becomes kT/hf, typically $\sim 600$ at ambient temperature at $10 \mathrm{GHz}$. This flux of photons becomes the familiar $k T B$ Watts of thermal radiation in microwave systems (which normally operate on a single mode) for a signal bandwidth of B. Formal analysis of this leads to the Johnson noise power of $4 k T B$ in electrical systems. If this thermal radiation is sampled satisfying the Nyquist theorem, a single sample in a time $(1 / 2 B)$ will capture on average the radiation from $2 k T / h f$ photons, which is in the region of 1200 for the proposed system. This is a very large number compared to the possible single entangled photon that is to be detected. 
Shot noise will also contribute, but like Johnson noise it will be random and it is assumed that the combined noise power be many orders of magnitude larger than the entangled photon flux power. It is normal in circumstances, when signals are very much smaller than the noise, to use some form of signal integration technique to increase the signal to noise ratio over a period of time. The improvement which results is normally proportional to the root of the integration time.

A proposed circuit that may enable a coherent integration of a flux of entangled photons is shown in Figure 1 and the envisaged spectra of the entangled photons in the different sections of this circuit are shown in Figure 2. The idea is to reproduce in the millimetre wave band that which has been done in the optical for creating entangled photons, by using radio frequency counter parts of optical devices. Coherent pump radiation at $20 \mathrm{GHz}$ would be injected into a non-linear birefringent material. Signal and idler entangled photons generated by SPDC will travel in the two separate arms of the system. Waveplates and polarisers would be used to define states to be measured. The polarisation selectors, likely to be a waveguide horn to coaxial transitions, will select which polarisations will be measured by the system.

Thermal radiation also generated by the source and the surroundings, by virtue of their finite temperature, will also accompany the entangled photons and collectively these will be amplified by a phase insensitive (ie phase preserving) amplifier and then band-pass filtered before passing into an up-shifting (frequency summing) mixer. The mixer effectively multiplies the signal and idler together and outputs the product at the sum frequency, which is $20 \mathrm{GHz}$ in this case. This sum frequency is just that of the pump (a consequence of conservation of energy in Eq. 2). This means however the signal and idler are distributed in frequency, they will always combine at the pump frequency. Therefore the bandwidth over which entangled photons can be detected is maximised, as the system will be sensitive to both degenerate and non-degenerate entangled photons. This maximises the number of entangled photons that can be detected.

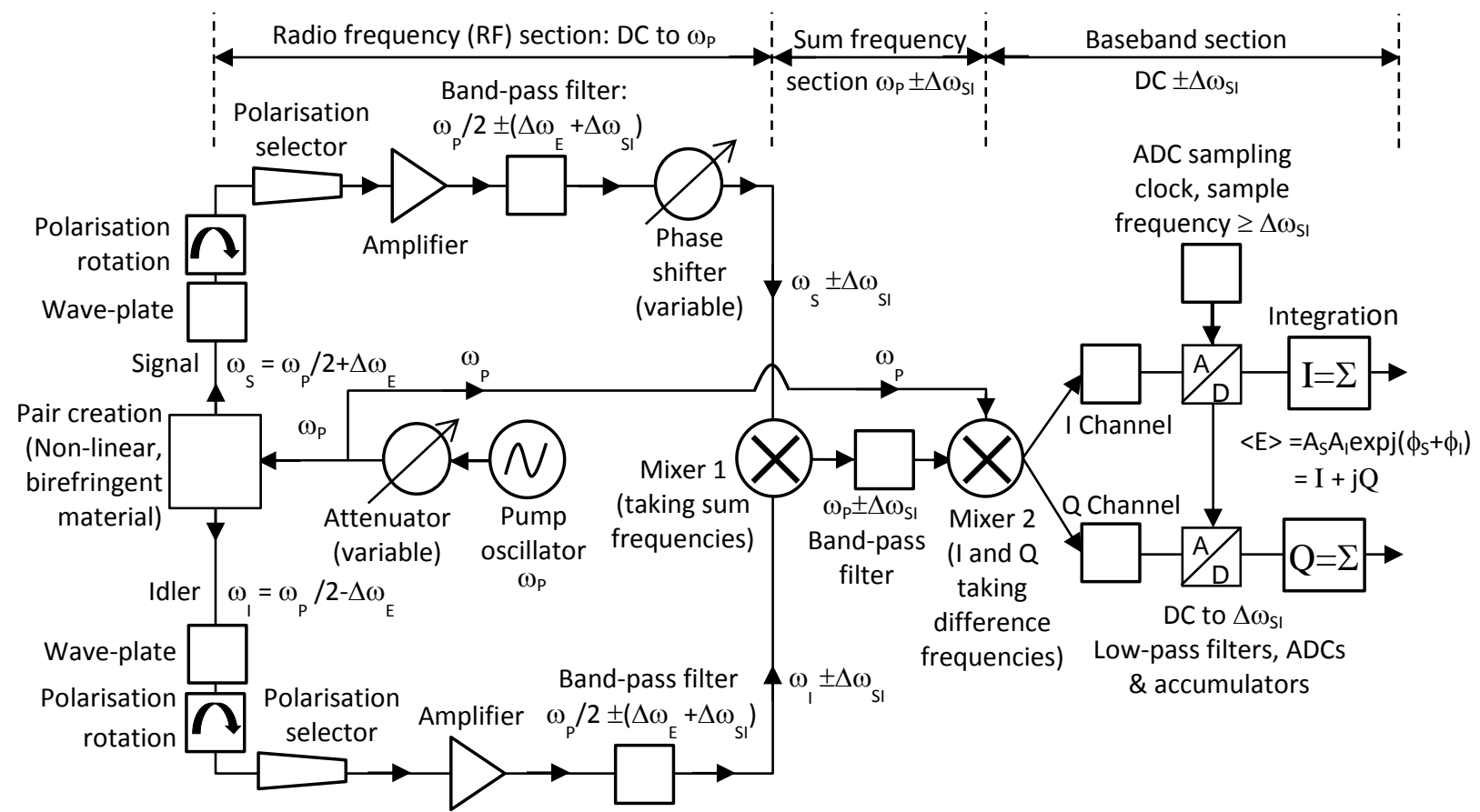

Figure 1. The millimetre wave single channel Bell Test circuit for entangled photon pair generation (via SPDC) and analysis in (using a homodyne interferometer) in the Rayleigh-Jean regime $(h f<k T)$.

The signature of the entangled photons has now been effectively modulated on to a carrier frequency which has the same frequency as the pump. Furthermore, as the phase of the signal and idler has been summed, the phase of the entanglement signature will be the same as that of the pump (a consequence of the conservation of momentum in Eq. 3) or just shifted by a constant phase offset due to different path lengths of the signal and idler. This then means that coherent detection can be used to integrate the entangled signatures from many entangled photon pairs to overcome the large amount of thermal noise in the system. A second mixer can be used to mix the entanglement signature at $20 \mathrm{GHz}$ with the pump frequency also at $20 \mathrm{GHz}$, to bring the entanglement signature down to baseband. This mixer can be of the 
in-phase (I) and quadrature (Q) down-shifting mixer type; this generates the complex amplitude directly at baseband and these outputs can be sampled by two analogue to digital converters and the results store in two separate accumulators, one for I and one for Q. The fact that there is phase coherence between the pump and the entangled photons means that the signal stored in the accumulators will be a coherently integrated signal. As the signal and idler are effectively constructively interfering using a pump in the creation of the entangled pairs and then in their detection, by mixing down to baseband, the system can justifiably be referred to as a homodyne interferometer.

The amplitude associated with the entangled photons as they propagate through the different sections of the system can be summarised as follows:

1) Radio frequency (RF) section: Signal $(t)=A_{S} \exp j\left(\omega_{S} t+\phi_{S}\right)$;

2) Sum frequency section: Signal $\times \operatorname{Idler}(t)=A_{S} A_{I} \exp j\left(\left[\omega_{S}+\omega_{I}\right] t+\phi_{S}+\phi_{I}\right)$

3) Baseband section: $\quad$ Signal $\times \operatorname{Idler}(t)=A_{S} A_{I} \exp j\left(\phi_{S}+\phi_{I}\right) \equiv A_{S} A_{I} \exp j \phi_{P}$

where $\mathrm{A}, \omega$ and $\phi$ refer to the amplitude, angular frequency and phase, and the subscripts $\mathrm{P}, \mathrm{S}$ and I refer to the pump, the signal and the idler respectively. The principles of conservation of energy and momentum given in Eq.2 and Eq.3 enable the coherent integration of many pairs of entangled photons, so that a Bell Test can be realised in the presence of the large number of thermally generated photons in the Rayleigh-Jean regime.

It might appear from the above that this may only be a system that could detect the phase of the pump. However, this can only happen if that phase information is transferred via the entangled photon pair; there is no classical process that can transfer this information.

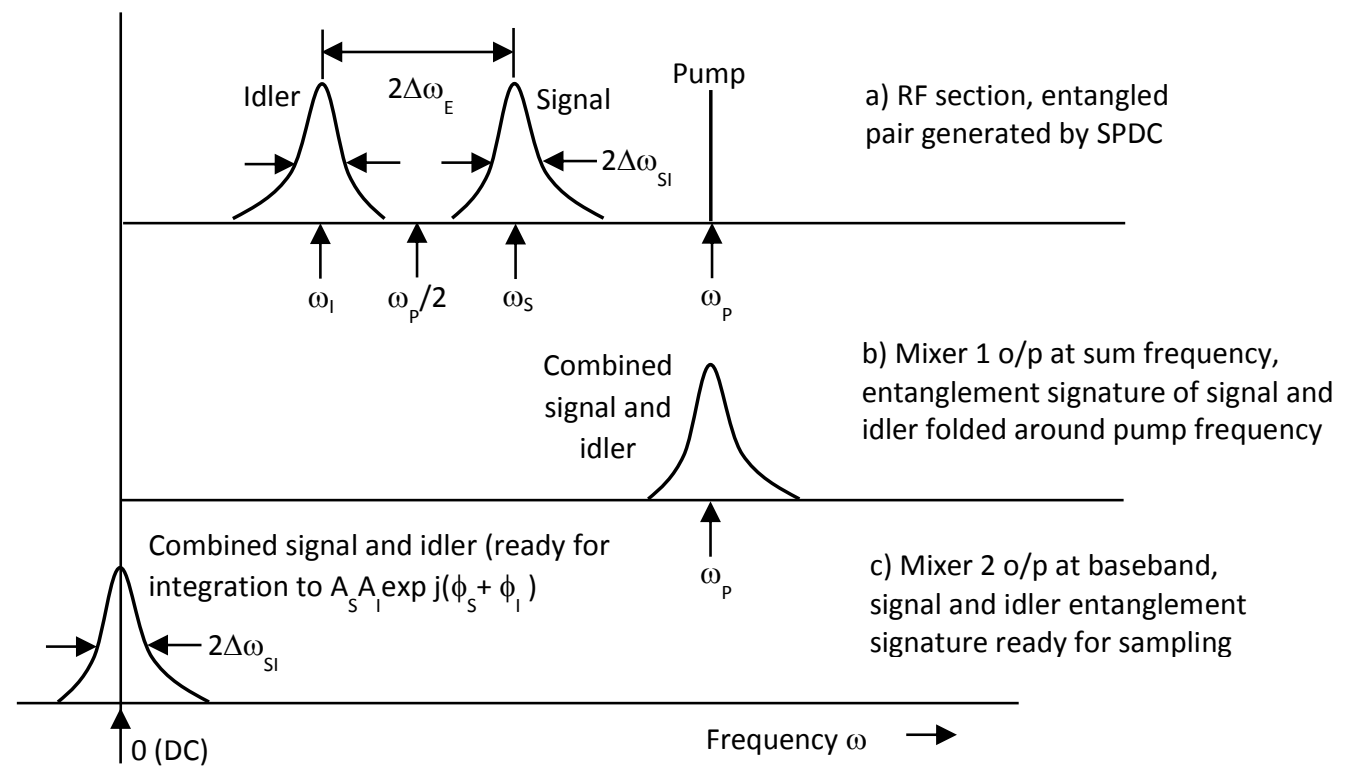

Figure 2. The spectra of the entangled photons in a) the RF section, b) at the sum frequencies (output of mixer 1) and c) at baseband section (output of mixer 2) of the millimetre wave Bell Test of Figure 1.

As the noise power in the system generated by the thermal radiation and the electronic noise is likely to be much greater than the power of the entangled photon flux, the entangled signature will be unobservable on a single sample. However, as the noise in the signal and idler channels will be uncorrelated, the long term average of its amplitude will be zero. This means that the long term average of the output from the interferometer will only contain the complex amplitude of the entangled photon pair. The real and imaginary parts of the complex amplitude of the entanglement signature will be contained in the I and Q accumulation registers.

It is assumed in the above that the frequencies of the signal and idler photons are distributed in frequency $\pm \Delta \omega_{\mathrm{E}}$ about half the pump frequency $\omega_{\mathrm{P}} / 2$, and that the half width in frequency space of signal and idler photons are both $\Delta \omega_{\mathrm{SI}}$. This means if bandpass filters are placed in the signal and idler arms of the interferometer centred on the frequency of $\omega_{\mathrm{p}} / 2$ 
having a passband of $\pm\left(\Delta \omega_{\mathrm{E}}+\Delta \omega_{\mathrm{SI}}\right)$, photons of these frequencies will be selected for transmission to the first mixer. After the first mixer there can be a further bandpass filter centred on $\omega_{\mathrm{P}}$ with a passband of $\pm \Delta \omega_{\mathrm{SI}}$ and this will prevent unwanted frequencies from entering the second mixer. After the second mixer there can be a DC to $\Delta \omega_{\mathrm{SI}}$ low-pass filters in the I and Q channels which act as anti-aliasing filters prior to sampling. If in the I and Q channels the high frequency cut-offs of the low-pass filters are set to $\Delta \omega_{\mathrm{SI}}$ and the analogue to digital converters in each channel sample at a frequency of $2 \Delta \omega_{\mathrm{SI}}$ the sampling of the photons will satisfy the Nyquist criterion. If for example it was assumed that the bandwidth of a photon was $2 \mathrm{GHz}$ the ADCs would be sampling at a total frequency of $4 \mathrm{GHz}$. Such a high sampling frequency sounds challenging, but it can be achieved relatively easily if single bit sampling is performed using clocked comparators to make the sample [17]. Signals could then enter the serial input of a Field Programmable Gate Array, where the accumulation of the I and Q signature could be performed digitally.

The sampling in the circuit of Figure 1 is performed synchronously by a clock which is set to satisfy the Nyquist criterion for the photon bandwidth. This is quite different to the sampling in classical Bell Tests where thresholded coincidence counting at used. This different approach should not in principle cause a problem, because if the Nyquist sampling theorem criterion is satisfied, all information is captured.

The general architecture in Figure 1 and the technology that would be used are very similar to that used in aperture synthesis imaging systems from radio astronomy, which used mixers, filters, high-speed (Giga sample per second) short word ( $\leq 4$ bits) sampling [16]. Typically these systems integrate between tens of millions and billions of samples (to overcome thermal photon noise) on multiple channels to create a single image, not only for radio astronomy but also for terrestrial imaging applications [17], [18]. Hardware from these areas is therefore reusable in the development of quantum homodyne interferometers as the basis of a Bell Test.

\subsection{Closing Bell Test loop holes}

There may be other ways of implementing the Bell Test based on the above homodyne interferometer where for example there is (single-bit) digitisation in the radio frequency section, so that mixer 1 and mixer 2 can perform their respective actions of sum-frequency mixing followed by down-conversion to based band digitally. This would mean (single-bit) digital samples would be made separately in the signal and idler channels, before these were brought together in a mixer, thereby closing one of the Bell Test loop holes. The circuit of Figure 1 would represent the lowest cost and risk solution for initial investigations and it could be built using commercially available off-the-shelf components now.

\subsection{Varying the flux of entangled photons}

The pump source shown in Figure 1 used to generate the entangled photons has an attenuator directly in front of it. The purpose of this is to vary the number of entangled photons generated. This might be used in experiments to determine the link between the magnitude of the complex amplitude of the entangled photons and the power of the pump. If the generation of entangled photons in the SPDC is linked to a non-linear behaviour, it might be expected that there would be a non-linear relationship between the pump power and the magnitude of the entangled photon signature.

\subsection{Varying the phase in the entangled photon signal channel}

A useful experiment that could be carried out to validate the existence of entangled photons would be to vary the phase in say the signal channel and this is the reason for the variable phase shifter in Figure 1. Any phase variation here would be directly reproduced as a phase shift in the complex amplitude of the entangled signature in the Argand diagram. Since the only link between baseband complex amplitude and the entangled signal photon is the conservation of momentum a matching phase shift would confirm the existence of entangled photons.

\subsection{Determination of the single channel Bell Test Statistic ' $S$ '}

To determine if entangled photons in the Bell Test circuit of Figure 1 have infringed Bell's inequality it is necessary to determine the 'test statistic S' and see if this is greater than zero. This is normally done [8] by measuring the number of coincidence counts when the polarisation rotators are positioned at the Bell Angles, namely at $0^{\circ}, 45^{\circ}, 22.5^{\circ}$ and $67.5^{\circ}$, and also when there are no polarisers in the system, so as to maximise the difference between the quantum and the classical result. The polarisation rotators in Figure 1 are used for this purpose. In the optical Bell Test the Test Statistic ' $\mathrm{S}$ ' is normally determine from the number of coincidence counts. In the homodyne interferometer proposed here this quantity would be replaced by the complex square, $\mid$ Signal $\times$ Idler $\left.\right|^{2}$ of the integrated baseband signal and idler measurement, as this would be seen equivalent to the coincidence count. 


\subsection{Generating all Bell states}

The half-wave plate shown in the signal arm of the Bell Test circuit of Figure 1 is there so that all four Bell States can be created. By arranging the half-wave plate with it fast axis parallel and at $45^{\circ}$ to one of the linear polarisations all four Bell States can be created. This is possible because the half-wave plate in these orientations can change the sign of a single polarisation or change one linear polarisation to its orthogonal linear polarisation, eg. flip vertical to horizontal polarisation. A quarter-wave plate would be used to convert linear to circular polarisation, so measurements in the circular polarisation basis could be made.

\subsection{A two-channel Bell Test}

If the rotatable polarisers of Figure 1 are replace by rotatable polarising beam-splitters and an extra two receiver channels are used a two-channel Bell Test could be constructed. The 'test statistic' $S$ of this circuit must be greater than two to infringe the Bell inequality.

\subsection{More than one entangled photon in a sample}

If there is more than one entangled photon in a sample, then this should just increase the amplitude of the entangled signature. This is because the entangled photons retain the phase information via the conservation of momentum (Eq.3) and constructive interference will just generate a larger entanglement signature. It is uncertain if this situation would arise, but there is a variable attenuator on the output of the pump which could be used in the measurement of this dependency.

\section{SIMULATION OF THE AMBIENT TEMPERATURE MM-WAVE BELL TEST}

A semi-classical simulation of the generation and the analysis of entangled photons using the coherent detection method has been made. A flux of entangled photons has been created which is equal to the product of the efficiency of entangled photon pair creation and the number of pump photons.

The number of pump photons has been taken to the power of the pump, which has been taken to be $50 \mathrm{~mW}$ multiplied by a probability of entangled pair generation, taken to be pessimistically low at $10^{-11}$, divided by the pump photon energy $h f_{P}$, where $f_{P}$ is $20 \mathrm{GHz}$. Currently the entangled pair generation probability is unknown, but in the optical domain, the probability of entangled pair generation is in the region of $10^{-6}$, which is higher than in the original optical band experiments, due to the increase in efficiencies arising from phase matching in the non-linear crystal material [7].

The number of thermal photons in a sample time is just the ratio of the thermal power $(k T B)$, divided by the photon energy $h f_{S I}$ and multiplied by the sample time, which is $1 / 2 B$ to satisfy the Nyquist criterion. The number of thermal photons in a sample is therefore $k T / 2 h f_{S I}$. Assuming the entangled photons to be around a frequency $f_{S I}$ of $10 \mathrm{GHz}$, the number of thermal photons in a sample time becomes on average $\sim 300$.

The noise in the receiver section is modelled as a normal Gaussian distribution on the in-phase and quadrature components in the signal and idler channels, using four random numbers for each time sample. The signal and idler are modelled as harmonic, with a phase link to that of the pump (Eq. 3), but for successive samples in time there is no phase relation. This is achieved by adding random numbers uniformly distributed in phase between 0 and $2 \pi$ to all signal samples then subtracting the same random numbers from the idler samples. This means phase on the signal and idler will be random, simulating quantum randomness, but their sum will equal that of the pump. All random numbers are uncorrelated.

The amplitude of the entangled signature is taken to be unity and the noise is just taken to be the ratio of the number of thermal photons to the number of entangled photons. The time sequence of the signal and idler with their added Gaussian noise are then multiplied together to simulate the action of the first mixer. This generates a product at the frequency of that of the pump. The time sequence of this product is then multiplied by the time sequence of the pump, which brings the signal down to baseband.

On a single sample the noise at the baseband is massively greater than the entangled signal. However, by adding together many samples the entanglement signature can be recovered. For the simulation a sample time of 2 ns has been taken which puts the receiver bandwidth at $250 \mathrm{MHz}$. A total of $10^{5}$ samples have been integrated which at the $2 \mathrm{~ns}$ sample time would take $0.2 \mathrm{~ms}$ to acquire. A phase offset of $70^{\circ}$ has been included in the simulation, which is something that might arise due to the difference in path lengths between the signal and idler. This value could also be adjusted using the 
variable phase shifter in Figure 1. Plots of how the magnitude and phase of the complex mean of the output of the coherent integration vary as a function of the integration time is shown in Figure 3. The complex mean is taken to be that complex number that results from the individual means of the real values and the imaginary values. The magnitude and phase displayed in these figures is not the mean of the magnitudes and phases of these samples; these are different quantities.

It can be seen in Figure 3 that as the integration time increases, the coherently integrated magnitude and phase of the complex mean gets closer to the input values. After $10^{5}$ samples $(0.2 \mathrm{~ms})$ the magnitude at 1.00138155329 is close to the input value of unity, and the phase is found to be $69.2070418356^{\circ}$, which is close to the input value of $70^{\circ}$.
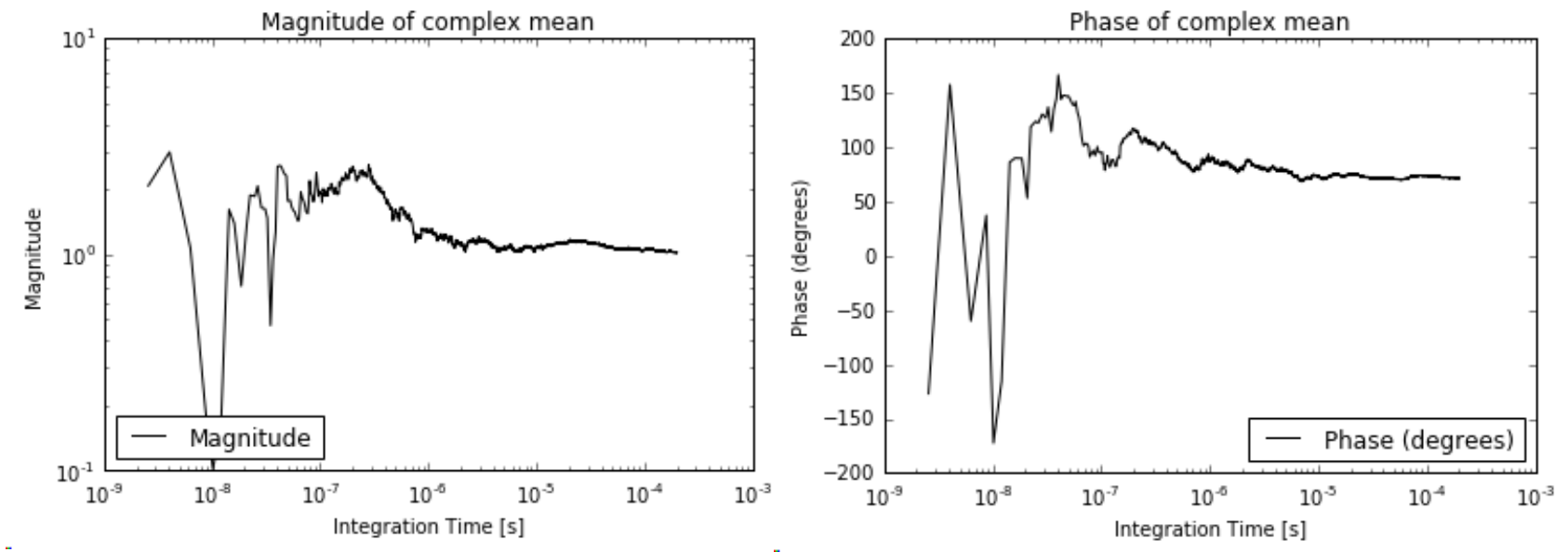

Figure 3. Coherent integration generates the magnitude (left) and phase (right) of the complex mean with a precision that increases with time, shown here for an integration time of up to $100 \mathrm{~ms}\left(10^{5}\right.$ samples $)$, for a pump power of $50 \mathrm{~mW}$ at $20 \mathrm{GHz}$, with the probability of entangled pair generation set to $10^{-11}$ per pump photon.

\section{RISK}

In proposing a novel circuit to enable a Bell Test in the Rayleigh-Jeans regime represent a shift away from the classical optical band experiments and cryogenically cooled millimetre wave which both operate in the quantum regime $(h f>k T)$. In making this step it is important to identify areas where there is risk, as these might be reasons why the proposed technique is flawed. Some of these risk areas are now identified and these could be explored in more detail when the circuit is built and tested.

\subsection{Correlated thermal emission swamps the entanglement signature}

The van Cittert-Zernike theorem [19] says that thermal emission from a point source will be correlated and the emission from an extended source will be partially correlated, this is the basis for the aperture synthesis imaging technique [17]. So if thermal emission from the non-linear birefringent material is correlated, this will be detected by the interferometer. However, the van Cittert-Zernike theorem also says that for the emission to be correlated it must have the same frequency. Therefore to remove any thermal emission that would be correlated a band-stop filter can be placed centred on $\omega_{p} / 2$ in the radio-frequency section (in the signal and idler arms) of the interferometer. This would however prevent the degenerate photons from being detected, but since the circuit is sensitive to non-degenerate photons, this would not prevent the system from working. In the course of experiments to demonstrate the circuit, the effects of using this filter on the interference could be investigated.

\subsection{Stimulated emission destroys the entanglement}

In the Rayleigh-Jean regime the ratio of stimulated emission to spontaneous emission is just kT/hf, which is $\sim 600$ for $10 \mathrm{GHz}$ at 290 Kelvin [12]. However, since the system is in equilibrium (as the system temperature is stable) then emission (dominantly stimulated) must be equal to the absorption. Since the proposed system would use low loss components and operate in either free space or waveguide, then by design absorption in components could easily be $\sim 1$ $\mathrm{dB}$ or less. This means absorption is likely to be $\leq 24 \%$, so this same fraction would represent the amount of stimulated emission. This means stimulated emission is only likely to destroy a small fraction $(\leq 24 \%)$ of entangled photons. 


\subsection{Amplification destroys the entanglement or adds unacceptable levels of noise}

'You can't clone a photon, so you can't amplify it' is a phrase that's often heard. However, in the case of amplification in Figure 1, what is happening here is that it is not increasing the amount of information, but raising the level of the electrical form of it, so it becomes larger than the noise from other components and can therefore be detected. This is very similar to what happens in the optical Bell Tests using SPADs. The difference here is that phase and amplitude need to be preserved. The concepts surrounding phase insensitive (sometime referred to as phase preserving) amplifiers is addressed by Caves [20]. It's claimed in this publication that the phase preserving amplifiers will add the noise power equivalent to at least half a photon of noise. This however, is a very small amount of noise compared to the noise from $\sim 300$ thermal photons that will accompany the entangled photon. Since the whole concept here is based on the coherent integration, the noise power associated with half a photon is seen as relatively minimal.

\subsection{Entangled mm-wave photons won't be generated by the available non-linear birefringent materials}

There is no record of entangled photons being generated by millimetre wave sources at ambient temperature. This is perhaps a circular argument, as if there is no system to prove the existence of entangled photons in the Rayleigh-Jeans regime, then there will be no confirmation that such sources exist. Perhaps the non-linearity of the medium of the source will not be strong enough to generate a flux of entangled photons. Perhaps it may not be possible to separate the entangled photons into two streams, so they can enter the signal and idler arms of the analyser. However, from the theory there is no reason in principle why the generation of entangled photons in the millimetre wave band should be any different from the equivalent process taking place in the optical band. Considering the circuit of Figure 1, this may well turn out to be an opportunity to break the above circular argument by developing a system that can detect entangled photons in the Rayleigh-Jeans regime. This could then be used to identify sources of entangled photons in the millimetre wave band and investigate the applications that may follow.

\subsection{Absence of a rigorous quantum evaluation}

The approach presented here of the Bell Test circuit of Figure 1 is semi-classical. A full quantum analysis of the circuit is needed for a complete evaluation of the system. In the absence of the full quantum treatment, it may be possible that some fundamental aspect has not been considered that would make the circuit unworkable. A full quantum treatment would require the rigorous evaluation of the entanglement generation process, the amplification, the filtering, the mixing and the sampling. Considerations in these areas are now being made. Findings from these will be used to refine the above simulations.

\section{APPLICATIONS}

A system that can demonstrate the existence of entangled photons in the Rayleigh-Jeans regime would be used to investigate the phenomenon of entanglement in the millimetre wave band and the possible sources of entangled photons. This might include an investigation into whether biological systems have the potential to generate entangled photons, or if they exploit entanglement in any way. Considerations might also be made as to whether there would be any implications of a homodyne interferometer for ambient temperature quantum computers.

Being able to identify an entanglement signature that is below the level of natural noise processes (like the thermal generation of photons) offers a natural covert sensing and communication capability. The pump that creates the entangled photons is a key to identifying the entangled pairs, but with the level of the noise far higher than the entangled photon power, it would be impossible to detect the entangled photons, let alone extract any information from them. This might open new applications areas where communication and radar interrogation would be fundamentally undetectable. This might build on some of the full polarimetric millimetre wave radar being develop for the band $18 \mathrm{GHz}$ to $26 \mathrm{GHz}$ [21].

\section{CONCLUSIONS}

A circuit has been proposed for the generation and analysis of entangled millimetre wave photons working at ambient temperature; a system for the Rayleigh-Jean regime, where the photon energy $(h f)$ is very much smaller than the thermal energy. A simple semi-classical analysis of the system has been made, which indicates that the system operating as a homodyne interferometer could be used as the basis for a Bell test, as the long term coherent integration of entangled signatures from many pairs could overcome the problems associated the thermal generation of photons. A simple simulation shows the coherent integration to be effective in measuring the complex amplitude associated with the 
entangled pairs in the Argand diagram. A full quantum analysis of the circuit is required as derisking exercise. The proposed circuit could be built and tested using low cost commercially available off-the-shelf components. Successful demonstration of the homodyne interferometer of Figure 1 could open up a new field in the area of millimetre wave quantum optics.

\section{FUTURE WORK}

It is hoped that funding will be found so that further analysis of the circuit could be made with a view to building and testing the circuit. The simulation would be refined to model noise from commercially available components and estimate the probability of entangled pair generation from available non-linear materials, to determine whether the Bell Test Statistic ' $S$ ' can lead to violation of Bell's criterion for the proposed configuration in Figure 1. A successful circuit would then be used to explore sources of entangled photons in the millimetre wave band, achieve a better understanding of the entanglement phenomenon in this band and begin to demonstrate some of the applications.

\section{ACKNOWLEDGEMENTS}

The author gratefully acknowledges the financial support for this work which came from the EPSRC TERANET programme, managed and technically led by John Cunningham, Giles Davies, Edmund Linfield and Martyn Chamberlain from the School of Electronic and Electrical Engineering at the University of Leeds. The author would also like to thank David Andrews, formerly of Manchester Metropolitan University, for stimulating discussions on the quantum limits of amplifiers and possible quantum processes that may limit the performance of the homodyne interferometer.

\section{REFERENCES}

[1] A. Einstein, B. Podolsky, N. Rosen: Phys. Rev. 47, 777 (1935).

[2] J. S. Bell: Physics 1, 105 (1964); Rev. Mod. Phys. 38, 447 (1966).

[3] Clauser, J. F., Horne, M.A., Shimony, A., Holt, R.A., "Proposed experiment to test local hidden-variable theories", Phys. Rev. Lett., 23 (15): 880-4, (1969).

[4] A. Aspect, P. Grangier, G. Roger: Phys. Rev. Lett. 49, 91 (1982).

[5] A. Aspect, J. Dalibard, G. Roger: Phys. Rev. Lett. 49, 1804 (1982).

[6] J. G. Rarity, P. R. Tapster, E. Jakeman, T. Larchuk, R. A. Campos, M. C. Teich and B. E. A. Saleh, Phys. Rev. Lett., 65 (1990), 1348.

[7] Prutchi, D \& Prutchi, S.R., "Exploring Quantum Physics through Hands-on Projects”, Wiley, 2012.

[8] Garrison, J.C. \& Chiao, R. Y. “Quantum Optics”, Oxford University Press, 2008.

[9] Walls, D.F. Milburn, G. J., "Quantum Optics", $2^{\text {nd }}$ Ed., Springer, 2008.

[10] Agarwal, G. S., "Quantum Optics”, Cambridge University Press, 2013.

[11] Gerry C. \& Knight P., "Introductory Quantum Optics”, Cambridge University Press, 2005.

[12] Loudon, R., "The Quantum Theory of Light", 3 ${ }^{\text {rd }}$ Ed. 2000.

[13] Bouwmeester, D., Ekert, A. \& Zeilinger, A. (Ed..), “The Physics of Quantum Information”, Springer, 2000

[14] Eichler, C., Wallraff, A. et al, "Observation of Entanglement between Itinerant Microwave Photons and a Superconducting Qubit”, Phys. Rev. Lett. 109, 240501, 14 ${ }^{\text {th }}$ Dec. 2012.

[15] Flurin, E., Huard, B. et al, "Generating Entangled Microwave Radiation Over Two Transmission Lines", Phys. Rev. Lett. 109, 183901, $2^{\text {nd }}$ November 2012.

[16] Thomson, A. R., Moran, J.M. and Swenson, G. W., "Interferometry and Synthesis in Radio Astronomy", Wiley, 2004.

[17] Salmon, N. A. “3-D Radiometric Aperture Synthesis Imaging”, IEEE TMTT, vol. 63, issue 11, pp. 35793587, Nov. 2015.

[18] Salmon, N. A., Macpherson, R., Harvey, A., Hall, P., Hayward, S., Wilkinson, P., Taylor, C., "First video rate imagery from a 32-channel 22-GHz aperture synthesis passive millimetre wave imager", SPIE, vol. 8188, October, 2011.

[19] Born, M. \& Wolf., "Principles of Optics", $7^{\text {th }}$ Ed. Cambridge University Press, 2005.

[20] Caves, C. M. "Quantum limits on noise in linear amplifiers", Phys. Rev. D, 26, 1817-39, 1982.

[21] Blackhurst, E. \& Salmon, N. A., "Experimental determination and simulations of the Huynen target parameters for full polarimetric millimetre wave concealed weapon recognition, Vol 10800, Millimetre Wave and Terahertz Sensors and Technology, Berlin, Sept. 2018. 